\title{
OPTICAL SOLITONS WITH ANTI-CUBIC NONLINEARITY USING THREE INTEGRATION SCHEMES
}

\author{
Anwar Ja'afar Mohamad Jawad ${ }^{1}$, Mohammad Mirzazadeh ${ }^{2}$, \\ Qin Zhou ${ }^{3} \&$ Anjan Biswas ${ }^{4,5}$ \\ ${ }^{1}$ Department of Computer Engineering, \\ Al-Rafidain University College, Baghdad-00964, Iraq \\ ${ }^{2}$ Department of Engineering Sciences, \\ Faculty of Technology and Engineering, East of Guilan, \\ University of Guilan, P.C. 44891-63157, Rudsar-Vajargah, Iran \\ ${ }^{3}$ School of Electronics and Information Engineering, \\ Wuhan Donghu University, Wuhan, 430212, PR China \\ ${ }^{4}$ Department of Mathematics, Faculty of Science, \\ King Khalid University, PO Box 9004, Abha-61413, Saudi Arabia \\ ${ }^{5}$ Department of Mathematics and Statistics, \\ Tshwane University of Technology, Pretoria-0008, South Africa
}

\begin{abstract}
This paper employed three integration schemes to obtain soliton solutions in optical fibers with anticubic nonlinearity. These are traveling waves, tanh-coth scheme and finally the modified simple equation method. These yielded bright solitons, singular solitons, dark-singular combo solitons and other waves. The existence criteria for these solitons are presented. The paper concludes with a discussion on conservation laws.
\end{abstract}

Key Words: solitons; anti-cubic nonlinearity; integrability. 


\section{INTRODUCTION}

Optical solitons with non-Kerr law nonlinearities is a growing area of research in nonlinear photonics [1-6]. There are several forms of such nonlinearities that has been studied during the past couple of decades. These are power law, parabolic law, dual-power law, polynomial law, triple-power law, saturable law. Apart from these laws that are frequently vsible in various papers, a couple of newly reported non-Kerr laws have attracted attention lately. These are quadratic-cubic law and anti-cubic law [2-6]. A few results have been reported with these two laws, particlarly with anti-cubic law $[2,5]$. This paper thus carries out a deeper investigation of this law, using three integration schemes, and will obtain bright, singular and dark-singular combo soliton solutions. The appropriate constraint conditions, for the existence of these solitons, are also presented. The integration algorithms adopted in this paper are traveling waves, tanh-coth method and finally the modified simple equation scheme that led to a new form of wave that is being reported for the first time in this paper. Finally, the conservation laws are discussed with the form of bright soliton solution obtained in this paper.

\section{TRAVELING WAVE SOLUTION}

Consider the nonlinear evolution equation (NLEE) in the form

$$
F\left(u, u_{t}, u_{x}, u_{y}, u_{t t}, u_{x x}, u_{x y}, u_{y y}, \ldots\right)=0
$$

where $u(x, y, t)$ is a traveling wave solution of equation (1). We use the transformation,

$$
u(x, y, t)=f(\xi)
$$

where $\xi=x+y-\lambda t$. This leads to the following changes:

$$
\frac{\partial}{\partial t}(.)=-\lambda \frac{\partial}{\partial \xi}(.), \quad \frac{\partial}{\partial x}(.)=\frac{\partial}{\partial \xi}(.), \frac{\partial}{\partial y}(.)=-\lambda \frac{\partial}{\partial \xi}(.), \ldots
$$

Using Eq. (3) to transfer the NLEE (1) to nonlinear ordinary differential equation (ODE)

$$
Q\left(f, f^{\prime}, f^{\prime \prime}, f^{\prime \prime \prime}, \ldots\right)=0 .
$$

The ODE, given by (4), is then integrated as long as all terms contain derivatives, where we neglect the integration constants.

\section{$2.1 \quad$ APPLICATION TO NLSE}

We investigate a cubic-quintic nonlinear Schrödinger's equation (NLSE) that with an additional anti-cubic nonlinear term, first introduced during 2003, and is of the form [1]

$$
i q_{t}+a q_{x x}+\left(b_{1}|q|^{-4}+b_{2}|q|^{2}+b_{3}|q|^{4}\right) q=0 .
$$

where $a, b_{1}, b_{2}$ and $b_{3}$ are real-valued constants. The independent variables are $x$ and $t$ that represents spatial and temporal co-ordinates. Again the dependent variable is $q(x, t)$ that is a complex-valued function. In (1), if $b_{1}=0$ it shrinks to NLSE with parabolic law or cubic-quintic law of nonlinearity that has been extensively studied in the past. It is this $b_{1}$ that formulates the anti-cubic nonlinear term.

Introduce the transformations

$$
q(x, t)=e^{i \theta(x, t)} u(\xi), \quad \theta=-\tau x+\omega t+\epsilon_{0}, \quad \xi=x-\lambda t+x_{0}
$$


where $\tau, \omega, \lambda, \epsilon_{0}$ and $x_{0}$ are real constants.

Substituting (6) into (5) and then breaking into real and imaginary parts gives a pair of relations. The imaginary part is:

$$
\lambda=-2 \tau a,
$$

while the real part implies:

$$
u^{\prime \prime}(\xi)-\frac{\left(\omega+a \tau^{2}\right)}{a} u(\xi)+\frac{b_{1}}{a} u^{-3}(\xi)+\frac{b_{2}}{a} u^{3}(\xi)+\frac{b_{3}}{a} u^{5}(\xi)=0 .
$$

Multiplying both sides of (8) by $u^{\prime}$ and integrating with respect to $\xi$, we get

$$
\frac{\left(u^{\prime}\right)^{2}}{2}-\frac{\left(\omega+a \tau^{2}\right)}{2 a} u^{2}-\frac{b_{1}}{2 a} u^{-2}(\xi)+\frac{b_{2}}{4 a} u^{4}+\frac{b_{3}}{6 a} u^{6}+b_{4}=0 .
$$

Thus,

$$
\left(u^{\prime}\right)^{2}-c_{0} u^{2}-c_{1} u^{-2}+c_{2} \frac{u^{4}}{2}+c_{3} \frac{u^{6}}{3}+c_{4}=0,
$$

where

$$
c_{0}=\frac{\left(\omega+a \tau^{2}\right)}{a}, \quad c_{1}=\frac{b_{1}}{a}, \quad c_{2}=\frac{b_{2}}{a}, \quad c_{3}=\frac{b_{3}}{a}, \quad c_{4}=2 b_{4} .
$$

Let $u^{2}=v$, then

$$
u^{\prime}=\frac{1}{2 u} v^{\prime}
$$

Substitute Eq.(12) in Eq. (10) gives:

$$
\left(v^{\prime}\right)^{2}-4 c_{0} v^{2}-4 c_{1}+2 c_{2} v^{3}+\frac{4}{3} c_{3} v^{4}+4 c_{4} v=0 .
$$

\subsection{UNDETERMINED COEFFICIENTS}

This section will utilize the principle of undetermined coefficients to secure bright, dark and singular 1-soliton solution to NLSE with anti-cubic nonlinearity. The starting point is a guess for the structure of these soliton solutions. This guess is substituted into NLSE. The undetermined coefficients of the linearly independent functions are set to zero which displays the parameter dynamics and the corresponding constraints that naturally emerge from the structure.

\subsubsection{SINGULAR SOLITONS}

The solutions of many nonlinear equations can be expressed in the form:

$$
f(\xi)=\sigma \operatorname{csch}^{\beta}(\mu \xi)
$$

Where $\sigma, \mu$ and $\beta$ are parameters to be determined, $\mu$ and $\lambda$ are the wave number and the wave speed, respectively. We use and their derivative.

$$
\begin{aligned}
& f^{\prime}(\xi)=-\sigma \beta \mu \operatorname{csch}^{\beta}(\mu \xi) \operatorname{coth}(\mu \xi), \\
& f^{\prime \prime}(\xi)=\sigma \beta \mu^{2}\left\{(\beta+1) \operatorname{csch}^{\beta+2}(\mu \xi)+\beta \operatorname{csch}^{\beta}(\mu \xi)\right\}, \\
& f^{\prime \prime \prime}(\xi)=-\sigma \beta \mu^{3}\left\{(\beta+1)(\beta+2) \operatorname{csch}^{\beta+2}(\mu \xi)+\beta^{2} \operatorname{csch}^{\beta}(\mu \xi)\right\} \operatorname{coth}(\mu \xi),
\end{aligned}
$$


and so on. We substitute (6) into the reduced equation (4), balance the terms of the sech functions and solve the resulting system of algebraic equations by using computerized symbolic packages. We next collect all terms with the same power in $\operatorname{csch}^{k}(\mu \xi)$ and set to zero their coefficients to get a system of algebraic equations among the unknown's $\alpha, \mu$ and $\beta$ and solve the subsequent system.

Seeking the solution by csch function method as in (14), Eq. (13) transforms to:

$$
\begin{aligned}
& \sigma^{2} \beta^{2} \mu^{2} \operatorname{csch}^{2 \beta+2}(\mu \xi)+\sigma^{2} \beta^{2} \mu^{2} \operatorname{csch}^{2 \beta}(\mu \xi)-4 c_{0} \sigma^{2} \operatorname{csch}^{2 \beta}(\mu \xi)-4 c_{1}+2 c_{2} \sigma^{3} \operatorname{csch}^{3 \beta}(\mu \xi) \\
& +\frac{4}{3} c_{3} \sigma^{4} \operatorname{csch}^{4 \beta}(\mu \xi)+4 c_{4} \sigma \operatorname{csch}^{\beta}(\mu \xi)=0 .
\end{aligned}
$$

Equating the exponents and the coefficients of each pair of the Csch functions we find the following algebraic system

$$
2 \beta+2=4 \beta \Leftrightarrow \beta=1 .
$$

Thus setting coefficients of Equations (16) to zero yields

$$
\begin{aligned}
& \mu^{2}+\frac{4}{3} c_{3} \sigma^{2}=0, \\
& \mu^{2}-4 c_{0}=0 .
\end{aligned}
$$

Solving the system of equations in (18) we get:

$$
\mu=2 \sqrt{\frac{\omega+a \tau^{2}}{a}}, \quad \sigma=\sqrt{-\frac{3\left(\omega+a \tau^{2}\right)}{b_{3}}},
$$

where

$$
a\left(\omega+a \tau^{2}\right)>0
$$

and

$$
b_{3}\left(\omega+a \tau^{2}\right)<0 .
$$

Then:

$$
u(x, t)=\sqrt{-\frac{3\left(\omega+a \tau^{2}\right)}{b_{3}}} \operatorname{csch}^{\frac{1}{2}}\left(2 \sqrt{\frac{\omega+a \tau^{2}}{a}}\left(x+2 \tau a t+x_{0}\right)\right),
$$

and finally

$$
q(x, t)=e^{i \theta(x, t)} \sqrt{-\frac{3\left(\omega+a \tau^{2}\right)}{b_{3}}} \operatorname{csch}^{\frac{1}{2}}\left(2 \sqrt{\frac{\omega+a \tau^{2}}{a}}\left(x+2 \tau a t+x_{0}\right)\right),
$$

where

$$
\theta=-\tau x+\omega t+\epsilon_{0}
$$

Thus, the singular 1-soliton solution given by (21) can be alternatively written as:

$$
q(x, t)=A \operatorname{csch}^{\frac{1}{2}}\left[B\left(x+2 \tau a t+x_{0}\right)\right] e^{i \theta(x, t)}
$$

where the free parameters $A$ and $B$ are given by

$$
A=\sqrt{-\frac{3\left(\omega+a \tau^{2}\right)}{b_{3}}}
$$

and

and the constraints are indicated above.

$$
B=2 \sqrt{\frac{\omega+a \tau^{2}}{a}}
$$




\subsubsection{BRIGHT SOLITONS}

The solutions of many nonlinear equations can be expressed in the form:

$$
f(\xi)=\sigma \operatorname{sech}^{\beta}(\mu \xi)
$$

Where $\sigma, \mu$ and $\beta$ are parameters to be determined, $\mu$ and $\lambda$ are the wave number and the wave speed, respectively. We use and their derivative.

$$
\begin{aligned}
& f^{\prime}(\xi)=-\sigma \beta \mu \operatorname{sech}^{\beta}(\mu \xi) \tanh (\mu \xi), \\
& f^{\prime \prime}(\xi)=-\sigma \beta \mu^{2}\left\{(\beta+1) \operatorname{sech}^{\beta+2}(\mu \xi)-\beta \operatorname{sech}^{\beta}(\mu \xi)\right\}, \\
& f^{\prime \prime \prime}(\xi)=\sigma \beta \mu^{3}\left\{(\beta+1)(\beta+2) \operatorname{sech}^{\beta+2}(\mu \xi)-\beta^{2} \operatorname{sech}^{\beta}(\mu \xi)\right\} \tanh (\mu \xi) .
\end{aligned}
$$

Seeking the solution by sech function method as in (23), Eq. (13) yields:

$$
\begin{aligned}
& \sigma^{2} \beta^{2} \mu^{2} \operatorname{sech}^{2 \beta}(\mu \xi)-\sigma^{2} \beta^{2} \mu^{2} \operatorname{sech}^{2 \beta+2}(\mu \xi)-4 c_{0} \sigma^{2} \operatorname{sech}^{2 \beta}(\mu \xi)-4 c_{1}+2 c_{2} \sigma^{3} \operatorname{sech}^{3 \beta}(\mu \xi) \\
& +\frac{4}{3} c_{3} \sigma^{4} \operatorname{sech}^{4 \beta}(\mu \xi)+4 c_{4} \sigma \operatorname{sech}^{\beta}(\mu \xi)=0 .
\end{aligned}
$$

Equating the exponents and the coefficients of each pair of the sech functions we find the following algebraic system

$$
2 \beta+2=4 \beta \Leftrightarrow \beta=1 .
$$

Thus setting coefficients of Equations (24) to zero yields

$$
\begin{aligned}
& -\mu^{2}+\frac{4}{3} c_{3} \sigma^{2}=0, \\
& \mu^{2}-4 c_{0}=0 .
\end{aligned}
$$

Solving the system of equations in (26) we get:

$$
\mu=2 \sqrt{\frac{\omega+a \tau^{2}}{a}}, \quad \sigma=\sqrt{\frac{3\left(\omega+a \tau^{2}\right)}{b_{3}}},
$$

where

$$
a\left(\omega+a \tau^{2}\right)>0
$$

and

$$
b_{3}\left(\omega+a \tau^{2}\right)>0 .
$$

Therefore,

$$
u(x, t)=\sqrt{\frac{3\left(\omega+a \tau^{2}\right)}{b_{3}}} \operatorname{sech}^{\frac{1}{2}}\left(2 \sqrt{\frac{\omega+a \tau^{2}}{a}}\left(x+2 \tau a t+x_{0}\right)\right),
$$

finally

$$
q(x, t)=e^{i \theta(x, t)} \sqrt{\frac{3\left(\omega+a \tau^{2}\right)}{b_{3}}} \operatorname{sech}^{\frac{1}{2}}\left(2 \sqrt{\frac{\omega+a \tau^{2}}{a}}\left(x+2 \tau a t+x_{0}\right)\right),
$$

where

$$
\theta=-\tau x+\omega t+\epsilon_{0} .
$$


Hence, the bright 1-soliton solution given by (21) can be alternatively written as:

$$
q(x, t)=A \operatorname{sech}^{\frac{1}{2}}\left[B\left(x+2 \tau a t+x_{0}\right)\right] e^{i \theta(x, t)}
$$

where, the amplitude $A$ and the inverse width $B$ are given by

$$
A=\sqrt{\frac{3\left(\omega+a \tau^{2}\right)}{b_{3}}}
$$

and

$$
B=2 \sqrt{\frac{\omega+a \tau^{2}}{a}}
$$

and the constraints are presented above.

\section{TANH-COTH METHOD}

The key step is to introduce the new independent variable

$$
Y=\tanh (\xi),
$$

where $u(x, t)=U(\xi)$. This leads to the change of variables:

$$
\begin{aligned}
& \frac{d U}{d \xi}=\left(1-Y^{2}\right) \frac{d U}{d Y} \\
& \frac{d^{2} U}{d \xi^{2}}=-2 Y\left(1-Y^{2}\right) \frac{d U}{d Y}+\left(1-Y^{2}\right)^{2} \frac{d^{2} U}{d Y^{2}}, \\
& \frac{d^{3} U}{d \xi^{3}}=2\left(1-Y^{2}\right)\left(3 Y^{2}-1\right) \frac{d U}{d Y}-6 Y\left(1-Y^{2}\right)^{2} \frac{d^{2} U}{d Y^{2}}+\left(1-Y^{2}\right)^{3} \frac{d^{3} U}{d Y^{3}} .
\end{aligned}
$$

The subsequent step is that the solution is expressed in the form

$$
U(\xi)=\sum_{k=0}^{m} a_{k} Y^{k}+\sum_{k=1}^{m} b_{k} Y^{-k}
$$

where the parameter $m$ can be found by balancing the highest-order linear term with the nonlinear terms in Eq. (13), and $a_{0}, a_{1}, \ldots, a_{m}, b_{1}, b_{2}, \ldots, b_{m}, \tau, \omega$ are to be determined. Substituting Eq. (32) into Eq. (13) will yield a set of algebraic equations for $a_{0}, a_{1}, \ldots, a_{m}, b_{1}, b_{2}, \ldots, b_{m}, \tau, \omega$ because all coefficients of have $Y$ to vanish. Having determined these parameters, knowing that $m$ is positive integer in most cases, and using Eq. (13) we obtain analytic solutions $u(x, t)$, in a closed form.

$$
\left(1-Y^{2}\right)^{2}\left(\frac{d V}{d Y}\right)^{2}-4 c_{0} V^{2}-4 c_{1}+2 c_{2} V^{3}+\frac{4}{3} c_{3} V^{4}+4 c_{4} V=0 .
$$

Now, to determine the parameter $m$, we balance the linear term of highest-order with the highest order nonlinear terms. So, in Eq. (33) we balance $V^{4}$ with $\left(\frac{d V}{d Y}\right)^{2}$, to obtain: $(m+1)^{2}=4 m$, then $m=1$. The tanh-coth method admits the use of the finite expansion for :

$$
\begin{aligned}
& V=a_{0}+a_{1} Y+b_{1} Y^{-1}, \\
& V^{\prime}=a_{1}-b_{1} Y^{-2} .
\end{aligned}
$$


Substituting (34) in (33), we get:

$$
\begin{aligned}
& \left(a_{1}^{2}-2 a_{1}^{2} Y^{2}+a_{1}^{2} Y^{4}\right)-2 a_{1} b_{1}\left(Y^{-2}-2+Y^{2}\right)+b_{1}^{2}\left(Y^{-4}-2 Y^{-2}+1\right) \\
& -4 c_{0}\left(a_{0}+2 a_{1} b_{1}+2 a_{0} a_{1} Y+a_{1}^{2} Y^{2}+2 a_{0} b_{1} Y^{-1}+b_{1}^{2} Y^{-2}\right) \\
& -4 c_{1}+2 c_{2}\left(a_{0}^{3}+3 a_{0}^{2} a_{1} Y+3 a_{0} a_{1}^{2} Y^{2}+a_{1}^{3} Y^{3}+3\left(a_{0}^{2} Y^{-1}+2 a_{0} a_{1}+a_{1}^{2} Y\right) b_{1}+3\left(a_{0} Y^{-2}+a_{1} Y^{-1}\right) b_{1}^{2}+b_{1}^{3} Y^{-3}\right) \\
& \frac{4}{3} c_{3}\left[a_{0}^{4}+4 a_{0}^{3} a_{1} Y+6 a_{0}^{2} a_{1}^{2} Y^{2}+4 a_{0} a_{1}^{3} Y^{3}+a_{1}^{4} Y^{4}+4\left(a_{0}^{3} Y^{-1}+3 a_{0}^{2} a_{1}+3 a_{0} a_{1}^{2} Y+a_{1}^{3} Y^{2}\right) b_{1}\right. \\
& \left.+6\left(a_{0}^{2} Y^{-2}+2 a_{0} a_{1} Y^{-1}+a_{1}^{2}\right) b_{1}^{2}+4\left(a_{0} Y^{-3}+a_{1} Y^{-2}\right) b_{1}^{3}+b_{1}^{4} Y^{-4}\right]+4 c_{4}\left(a_{0}+a_{1} Y+b_{1} Y^{-1}\right)=0 .
\end{aligned}
$$

Equating expressions at $Y^{k},(k=-4,-3,-2,-1,0,1,2,3,4)$ to zero we have the following system of equations:

$$
\begin{aligned}
& 1+\frac{4}{3} c_{3} a_{1}^{2}=0 \\
& 3 c_{2}+8 c_{3} a_{0}=0 \\
& a_{1}+b_{1}+2 c_{0} a_{1}-3 c_{2} a_{0} a_{1}-\frac{4}{3} c_{3}\left(3 a_{0}^{2} a_{1}+2 a_{1}^{2} b_{1}\right)=0 \\
& -4 c_{0} a_{0} a_{1}+3 c_{2}\left(a_{0}^{2} a_{1}+a_{1}^{2} b_{1}\right)+\frac{8}{3} c_{3}\left[a_{0}^{3} a_{1}+3 b_{1} a_{0} a_{1}^{2}\right]+2 c_{4} a_{1}=0 \\
& a_{1}^{2}+4 a_{1} b_{1}+b_{1}^{2}-4 c_{0}\left(a_{0}+2 a_{1} b_{1}\right)-4 c_{1}+2 c_{2}\left(a_{0}^{3}+6 a_{0} a_{1} b_{1}\right)+\frac{4}{3} c_{3}\left[a_{0}^{4}+12 a_{0}^{2} a_{1} b_{1}\right]+4 c_{4} a_{0}=0 \\
& -8 c_{0} a_{0} b_{1}+6 a_{0}^{2} b_{1} c_{2}+\frac{16}{3} c_{3} a_{0}^{3} b_{1}+4 c_{4} b_{1}=0 \\
& -2 a_{1} b_{1}-2 b_{1}^{2}-4 c_{0} b_{1}^{2}+\frac{16}{3} c_{3} a_{1} b_{1}^{3}=0 \\
& 3+4 c_{3} b_{1}^{2}=0
\end{aligned}
$$

Solving the system of equations (36) we get:

$$
\begin{aligned}
& a_{0}=\frac{4}{3 c_{2}}, \quad a_{1}=b_{1}=\frac{2}{c_{2}} \sqrt{\frac{2}{3}}, \quad c_{0}=-2, \quad c_{1}=-\frac{4}{3 c_{2}}\left(2+\frac{65}{9 c_{2}}\right), \\
& c_{3}=-\frac{9}{32} c_{2}^{2}, \quad c_{4}=-\frac{76}{9 c_{2}}
\end{aligned}
$$

Then :

$$
v(x, t)=\frac{2}{3 c_{2}}\left\{2+\sqrt{6}\left[\tanh \left(x+2 \tau a t+x_{0}\right)+\operatorname{coth}\left(x+2 \tau a t+x_{0}\right)\right]\right\}
$$

and

$$
u(x, t)=\left[\frac{2}{3 c_{2}}\left\{2+\sqrt{6}\left[\tanh \left(x+2 \tau a t+x_{0}\right)+\operatorname{coth}\left(x+2 \tau a t+x_{0}\right)\right]\right\}\right]^{\frac{1}{2}} .
$$

Therefore:

$$
q(x, t)=e^{i \theta(x, t)}\left[\frac{2}{3 c_{2}}\left\{2+\sqrt{6}\left[\tanh \left(x+2 \tau a t+x_{0}\right)+\operatorname{coth}\left(x+2 \tau a t+x_{0}\right)\right]\right\}\right]^{\frac{1}{2}},
$$

where

$$
\theta=-\tau x+\omega t+\epsilon_{0}
$$

Equation (40) represents a dark-singular combo soliton solution. 


\section{MODIFIED SIMPLE EQUATION METHOD}

We look for solutions of Eq. (13) in the form

$$
\begin{gathered}
v=A_{0}+A_{1} \frac{\psi_{\xi}}{\psi}, \\
v_{\xi}=A_{1}\left(\frac{\psi_{\xi \xi}}{\psi}-\frac{\psi_{\xi}^{2}}{\psi^{2}}\right) .
\end{gathered}
$$

Then

$$
\begin{aligned}
& A_{1}^{2}\left(\frac{\psi_{\xi \xi}}{\psi}-\frac{\psi_{\xi}^{2}}{\psi^{2}}\right)^{2}-4 c_{0}\left(A_{0}+A_{1} \frac{\psi_{\xi}}{\psi}\right)^{2}+2 c_{2}\left(A_{0}+A_{1} \frac{\psi_{\xi}}{\psi}\right)^{3}+\frac{4}{3} c_{3}\left(A_{0}+A_{1} \frac{\psi_{\xi}}{\psi}\right)^{4} \\
& +4 c_{4}\left(A_{0}+A_{1} \frac{\psi_{\xi}}{\psi}\right)-4 c_{1}=0 .
\end{aligned}
$$

Then, we have

$$
\begin{aligned}
& A_{1}^{2}\left\{\frac{\psi_{\xi \xi}^{2}}{\psi^{2}}-2 \psi_{\xi \xi} \frac{\psi_{\xi}^{2}}{\psi^{3}}+\frac{\psi_{\xi}^{4}}{\psi^{4}}\right\}+\frac{4}{3} c_{3}\left\{A_{0}^{4}+4 A_{0}^{3} A_{1} \frac{\psi_{\xi}}{\psi}+6 A_{0}^{2} A_{1}^{2} \frac{\psi_{\xi}^{2}}{\psi^{2}}+4 A_{0} A_{1}^{3} \frac{\psi_{\xi}^{3}}{\psi^{3}}+A_{1}^{4} \frac{\psi_{\xi}^{4}}{\psi^{4}}\right\} \\
& +2 c_{2}\left\{A_{0}^{3}+3 A_{0}^{2} A_{1} \frac{\psi_{\xi}}{\psi}+3 A_{0} A_{1}^{2} \frac{\psi_{\xi}^{2}}{\psi^{2}}+A_{1}^{3} \frac{\psi_{\xi}^{3}}{\psi^{3}}\right\}-4 c_{0}\left\{A_{0}^{2}+2 A_{0} A_{1} \frac{\psi_{\xi}}{\psi}+A_{1}^{2} \frac{\psi_{\xi}^{2}}{\psi^{2}}\right\} \\
& +4 c_{4}\left\{A_{0}+A_{1} \frac{\psi_{\xi}}{\psi}\right\}-4 c_{1}=0 .
\end{aligned}
$$

Equating expressions at $\psi^{-1}, \psi^{-2}, \psi^{-3}$, and $\psi^{-4}$ to zero we have the following system of equations:

$$
\begin{aligned}
& \left(1+\frac{4}{3} c_{3} A_{1}^{2}\right) \psi_{\xi}^{4}=0, \\
& -\psi_{\xi \xi}+\frac{8}{3} c_{3} A_{0} A_{1} \psi_{\xi}+c_{2} A_{1} \psi_{\xi}=0, \\
& \psi_{\xi \xi}^{2}+\left(8 c_{3} A_{0}^{2}+6 c_{2} A_{0}-4 c_{0}\right) \psi_{\xi}^{2}=0, \\
& \left(\frac{8}{3} c_{3} A_{0}^{3}+3 A_{0}^{2} c_{2}-4 c_{0} A_{0}+2 c_{4}\right) A_{1} \psi_{\xi}-2 c_{1}=0, \\
& \left(\frac{2}{3} c_{3} A_{0}^{3}+c_{2} A_{0}^{2}-2 c_{0} A_{0}+2 c_{4}\right) A_{0}-2 c_{1}=0 .
\end{aligned}
$$

Solving the system of equations in (45):

$$
A_{0}=\frac{-3 c_{2} \mp \sqrt{26 c_{2}^{2}+96 c_{3} c_{0}}}{8 c_{3}} .
$$

Case A:

$$
\psi_{\xi}=A,
$$


where

$$
A=\frac{\left\{\frac{2}{3} c_{3} A_{0}^{3}+c_{2} A_{0}^{2}-2 c_{0} A_{0}+2 c_{4}\right\} A_{0}}{\left\{\frac{8}{3} c_{3} A_{0}^{3}+3 A_{0}^{2} c_{2}-4 c_{0} A_{0}+2 c_{4}\right\} A_{1}} .
$$

Then

$$
\begin{gathered}
\psi=A \xi+\varepsilon, \\
u(\xi)=\sqrt{A_{0}+\frac{A A_{1}}{A \xi+\varepsilon}}, \quad \xi=x+2 \tau a t+x_{0} .
\end{gathered}
$$

Thus, we have

$$
q(x, t)=e^{i \theta(x, t)} \sqrt{A_{0}+\frac{A A_{1}}{A\left(x+2 \tau a t+x_{0}\right)+\varepsilon}},
$$

where

$$
\theta=-\tau x+\omega t+\epsilon_{0}
$$

\section{Case B:}

$$
\psi_{\xi \xi}-B \psi_{\xi}=0,
$$

where

$$
B=A_{1}\left(\frac{8}{3} c_{3} A_{0}+c_{2}\right) .
$$

The solution of Eq.(52) is:

$$
\begin{gathered}
\psi=\alpha+\beta e^{B \xi}, \\
\psi_{\xi}=\beta B e^{B \xi} .
\end{gathered}
$$

Then

$$
u(\xi)=\sqrt{A_{0}+\frac{\beta B A_{1} e^{B \xi}}{\alpha+\beta e^{B \xi}}}, \quad \xi=x+2 \tau a t+x_{0} .
$$

Thus, we have

$$
q(x, t)=e^{i \theta(x, t)} \sqrt{A_{0}+\frac{\beta B A_{1} e^{B\left(x+2 \tau a t+x_{0}\right)}}{\alpha+\beta e^{B\left(x+2 \tau a t+x_{0}\right)}}},
$$

where

$$
\theta=-\tau x+\omega t+\epsilon_{0} .
$$




\section{CONSERVATION LAWS}

The bright 1-soliton solution of (5) is rewritten in a compact form as:

$$
q(x, t)=A \sqrt{\operatorname{sech}\left[B\left(x-v t+x_{0}\right)\right]} e^{i\left(-\kappa x+\omega t+\theta_{0}\right)}
$$

Here, $A$ is the amplitude of the soliton, $B$ is its inverse width, $v$ is the speed and $x_{0}$ is the center position. From the phase component, $\kappa$ is the soliton frequency, $\omega$ is the wave number and finally $\theta_{0}$ is the phase constant.

Now, the three conserved quantities are power $(P)$, linear momentum $(M)$ and the Hamiltonian $(H)$ that are respectively given as

$$
\begin{gathered}
P=\int_{-\infty}^{\infty}|q|^{2} d x=\frac{\pi A^{2}}{B} \\
M=i a \int_{-\infty}^{\infty}\left(q q_{x}^{*}-q^{*} q_{x}\right) d x=\frac{a \kappa \pi A^{2}}{B},
\end{gathered}
$$

and

$$
\begin{aligned}
H & =\int_{-\infty}^{\infty}\left(a\left|q_{x}\right|^{2}-\frac{b_{1}}{|q|}+\frac{b_{2}|q|^{4}}{2}+\frac{b_{3}|q|^{6}}{3}\right) d x \\
& =\frac{A^{2}}{24 B}\left(3 \pi a B^{2}+24 b_{2} A^{2}+4 \pi b_{3} A^{4}\right)-\frac{b_{1}}{A B} \int_{-\infty}^{\infty} \sqrt{\cosh s} d s
\end{aligned}
$$

Thus, the Hamiltonian is in terms of quadratures and consequently must resort to numerics.

\section{CONCLUSIONS}

This paper studied solitons in optical fibers with anti-cubic nonlinearity by the aid of three integration schemes. The traveling wave hyypothesis coupled with the method of undetermined coefficients revealed bright and singular soliton solutions. The tanh-coth method obtained dark-singular combo solitons. Finally, the modified simple equation scheme retrieved new forms of waves that are being reported for the first time in this paper. The existence criteria for these waves and solitons, also known as constraint conditions, are listed. Finally, the paper gives the conservation laws from bright soliton solutions. It is observed that the Hamiltonian of the solitons do not exist because of a divergent integral.

The results of this paper pave way for additional research material. In future, other integration schemes will be implemented to extract further novel solutions to the governing NLSE. A few of these are $Q$-function scheme, Lie symmetry approach, exp-function method, $G^{\prime} / G$-expansion algorithm and others. The results of these research will be reported elsewhere. 


\section{References}

[1] A. Biswas \& S. Konar. Introduction to non-Kerr law optical solitons. CRC Press. Boca Raton FL. (2006).

[2] R. Fedele, H. Schamel, V. I. Karpman \& P. K. Shukla. "Envelope solitons of nonlinear Schrödinger equation with an anti-cubic nonlinearity". Journal of Physics A. Volume 36, Number 4, 1169-1173. (2003).

[3] J. Fujioka, E. Cortés, R. Pérez-Pascual, R. F. Rodríguez, A. Espinosa \& B. A. Malomed. "Chaotic solitons in the quadratic-cubic nonlinear Schrödinger equation under nonlinearity management". Chaos. Volume 21, 033120. (2011).

[4] X. Lü, W-X. Ma, J. Yu, F. Lin \& C. M. Khalique. "Envelope bright and dark soliton solutions for the Gerdjikov-Ivanov model". Nonlinear Dynamics. Volume 82, Issu3 3, 1211-1220. (2015).

[5] H. Triki, A. H. Kara, A. Biswas, S. P. Moshokoa \& M. Belic. "Optical solitons and conservation laws with anti-cubic nonlinearity". Optik. Volume 127, 12056-12062. (2016).

[6] H. Triki, A. Biswas, S. P. Moshokoa \& M. Belic. "Optical solitons and conservation laws with quadraticcubic nonlinearity". Optik. Volume 128, 63-70. (2017). 